\title{
Malaria vaccines targeting the pre-erythrocytic stage: a
}

\section{scoping review [version 1; peer review: 2 not approved]}

\author{
Teresa Ogeto1, Ferdinand Ndubi2, Mary Murithi (D)3, Richard Kagia(D) , \\ Esbon Wambugu(iD4, Titus Suge (iD) 4, Carolyne Chepkirui (iD) 5 , Josephat Tonui ${ }^{\text {, }}$ \\ Fiona Maiyo', Lydia Momanyi ${ }^{6}$, Michael Walekhwa (iD)
}

\author{
${ }^{1}$ Department of Biomedical Sciences, Kabarak University, Nakuru, Kenya, 20157, Kenya \\ 2Department of Pharmaceutical Chemistry \& Pharmaceutics, Kabarak University, Nakuru, Kenya, 20157, Kenya \\ ${ }^{3}$ Department of Pre-Clinical, Kabarak University, Nakuru, Kenya, 20157, Kenya \\ 4Pharmacology \& Pharmacognosy, Kabarak University, Nakuru, Kenya, 20157, Kenya \\ ${ }^{5}$ Department of Physical \& Biological Sciences, Kabarak University, Nakuru, Kenya, 20157, Kenya \\ ${ }^{6}$ Department of Pharmacy, Nakuru Level V Hospital, Nakuru, Kenya, 20157, Kenya
}

V1 First published: $07 \mathrm{Jul} 2020,9: 680$

https://doi.org/10.12688/f1000research.24320.1

Latest published: $07 \mathrm{Jul}$ 2020, 9:680

https://doi.org/10.12688/f1000research.24320.1

\section{Abstract}

Malaria is a deadly infectious parasitic disease that causes devastating morbidity and mortality globally. Despite being a public health concern, an effective vaccine for prevention of the disease remains elusive. Global efforts are exploring possible ways of developing and improving vaccines to counteract the complex nature in which Plasmodium falciparum evades the immune system. A number of vaccines have been developed in the past targeting the various parasitic life cycle stages. Transmission blocker vaccines, such as PpPf $S 25$, target the parasite stages in the mosquito vector. However, these herd vaccines only protect the immunized population. Vaccines targeting blood-stage forms, such as the AMA-1 and MSP-1 vaccines, are challenged by the complex metabolic pathways of erythrocytes and merozoites. Vaccines targeting the pre-erythrocytic sporozoite stage remain the most promising approach thus far. Here, we systematically review the literature on pre-erythrocytic stage vaccines and on-going work in the field. Furthermore, we highlight gaps in current knowledge and point to potential areas of future work. Articles on pre-erythrocytic malaria vaccines were obtained from Google scholar, PubMed and Cochrane starting from the year 2010. Ten papers were reviewed. A number of vaccines were reviewed highlighting; the vaccine type, clinical phase of trial, population demographics, vaccine immunogenicity, efficacy and safety. The RTS,S vaccine is reportedly the most advanced, having been rolled out for phase III clinical trials in a number of malaria-endemic African countries. The pre-erythrocytic vaccines discussed have made milestones in clinical trials. Some of the challenges elicited may be addressed via screening for novel antigens, exploring suitable vaccine

\section{Open Peer Review \\ Approval Status $\mathrm{X} X$ \\ 1 2 \\ version 1 \\ 07 Jul 2020

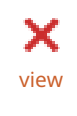 \\ X \\ view \\ 1. James M Burns Jr, Center for Molecular Parasitology, Drexel University College of Medicine, Philadelphia, USA \\ 2. Miguel Prudêncio ID , University of Lisboa, Lisboa, Portugal}

Any reports and responses or comments on the article can be found at the end of the article. 
administration vehicles, as well as using a combined multi-stage

vaccine approach.

\section{Keywords}

Malaria, Pre-erythrocytic, Plasmodium falciparum, Vaccines,

Sporozoites, efficacy

Corresponding author: Michael Walekhwa (walekhwam@gmail.com)

Author roles: Ogeto T: Methodology, Writing - Original Draft Preparation; Ndubi F: Conceptualization, Writing - Original Draft Preparation; Murithi M: Conceptualization, Methodology, Writing - Original Draft Preparation; Kagia R: Conceptualization, Writing Original Draft Preparation; Wambugu E: Formal Analysis, Writing - Original Draft Preparation; Suge T: Resources, Writing - Review \& Editing; Chepkirui C: Conceptualization, Writing - Review \& Editing; Tonui J: Conceptualization, Formal Analysis, Writing - Original Draft Preparation; Maiyo F: Formal Analysis, Methodology, Writing - Review \& Editing; Momanyi L: Conceptualization, Resources, Writing Review \& Editing; Walekhwa M: Conceptualization, Project Administration, Writing - Original Draft Preparation, Writing - Review \& Editing

Competing interests: No competing interests were disclosed.

Grant information: The author(s) declared that no grants were involved in supporting this work.

Copyright: ( 2020 Ogeto T et al. This is an open access article distributed under the terms of the Creative Commons Attribution License, which permits unrestricted use, distribution, and reproduction in any medium, provided the original work is properly cited.

How to cite this article: Ogeto T, Ndubi F, Murithi M et al. Malaria vaccines targeting the pre-erythrocytic stage: a scoping review [version 1; peer review: 2 not approved] F1000Research 2020, 9:680 https://doi.org/10.12688/f1000research.24320.1

First published: 07 Jul 2020, 9:680 https://doi.org/10.12688/f1000research.24320.1 


\section{Introduction}

Malaria, a disease commonly found in the tropics and subtropics is caused by parasites of the genus Plasmodium whereby P. falciparum causes the greatest disease burden. In 2018, it was estimated to have caused 228 million cases globally, out of which 93\% (213 million) occurred in Africa ${ }^{1}$. In Kenya, malaria is a major public health concern causing 6.7 million cases and 4000 mortalities in children annually $y^{2,3}$. Various community and facility interventional strategies, such as insecticide treated mosquito nets, indoor residual spraying, repellents and chemotherapy have significantly reduced the disease burden over the last decade $^{4}$. However, these strategies are limited in their efficacy; hence elimination of the disease is unlikely without an effective vaccine ${ }^{5}$. Consequently, an effective vaccine conferring high protective efficacy is required to combat the disease.

To date, a number of vaccines have been developed and are at various stages of clinical trials. These vaccines target specific stage(s) of the parasite life cycle, such as pre-erythrocytic (liver-stage), erythrocytic (blood-stage) and gametocytes ${ }^{6}$. The pre-erythrocytic vaccines (RTS,S, PfSPZ, TRAP, PP) that target the sporozoites have proved to be the most efficacious of these ${ }^{7}$. Their superiority is founded primarily on the premise that the sporozoites harbour less antigens and are thus easily targeted by an appropriate vaccine. This results in reduced merozoite production thereby rendering the blood stage immune responses superior ${ }^{8}$. The pre-erythrocytic stage is however faced with the challenge of culturing sporozoites in vitro, making it difficult to explore novel antigens? .

The RTS,S vaccine, which has progressed to phase III clinical trials, has reportedly been found to be safe and immunogenic when tested in African children in malaria endemic regions such as Kenya and Tanzania ${ }^{10,11}$. However, trials of this vaccine done in Africa have revealed low efficacy levels. A study done by Olotu et al (2016), on children aged 5-17 months immunized with RTS,S/ASO1A vaccine (cases) and rabies vaccine (controls), showed efficacy of $27 \%$ against first episode of clinical malaria ${ }^{11}$. Radiation-attenuated sporozoites (RAS) delivered through the bite of an infected mosquito was $100 \%$ protective in humans ${ }^{9}$. An irradiated $P$. falciparum sporozoites (PfSPZ) vaccine administered via intravenous injection also resulted in $100 \%$ protection in humans ${ }^{12}$. Other pre-erythrocytic vaccines that have so far been tested in humans include the thrombospondin related anonymous protein (TRAP), multi-epitope thrombospondin related adhesion protein $(M E-T R A P)$ and polyprotein (PP) vaccines $^{13,14}$

Despite the numerous interventions towards eliminating malaria, the parasite remains elusive. Vaccines remain to be the only hope to combat the disease. Therefore, efforts to understand $P$. falciparum biology and cross-talk mechanisms repressing the host immune response to an active infection are continuously being sought. In this review, we focus on milestones made on the pre-erythrocytic malaria vaccines in the last decade. We further highlight the challenges, immunogenicity and efficacy of these vaccines. Studies included mainly the $R T S, S$ vaccine whose clinical trials were carried out in Africa, a malaria endemic region.

\section{Methods}

This review was done according to Preferred Reporting Items for Systematic Reviews and Meta-Analyses (PRISMA) ${ }^{15}$. Articles included were those that had the relevance of the subject of malaria vaccine.

\section{Sources and search strategy}

Google Scholar, Cochrane and PubMed were the sources of articles. The search terminologies used were 'pre-erythrocytic malaria vaccines' and 'malaria vaccines'. An advanced search strategy in Google Scholar was done to return articles with all the words 'pre-erythrocytic' and 'malaria vaccines' in their titles. The exact phrases captured in the article titles were 'preerythrocytic malaria vaccines' or 'malaria vaccines'. Article titles without the words 'review' or 'meta-analysis' or 'expert opinion' or 'models' dated between 2010-2020 were retrieved. The last search in the databases was done on $24^{\text {th }}$ April 2020.

\section{Eligibility criteria}

Clinical trials were the only article type considered; reviews, meta-analysis, expert opinions, in vitro studies, mathematical and computer modeling studies were excluded. Articles published in English in the last ten years (2010-2020) were considered since promising milestones have been achieved in the last 10 years.

\section{Selection of articles}

To identify the suitability of the article to be included, the titles, abstracts, year of publication and type of study were considered. The papers were screened for eligibility by two reviewers and disagreements were resolved by consensus.

\section{Data extraction}

Relevant data was extracted from the included studies based on the type of the vaccine, study design, the clinical trial phase, the mode of vaccine administration, efficacy and safety profile, as well as related adverse events. Other important relevant information, such as the experimental procedures, were also evaluated for each study.

\section{Results}

In total, 17 articles were obtained from Google scholar, 7 from Cochrane and 231 from PubMed. After duplicate removal and screening, 10 articles were considered as meeting the criteria (Figure 1).

Table 1 exhibits the characteristics of the 10 articles that were included.

\section{Discussion}

The $R T S, S$ vaccine is by far the most advanced malaria vaccine in clinical development today. The vaccine confers its protective effect by targeting the circumsporozoite protein (CSP), a specific protein expressed by parasites in the pre-erythrocytic stage of the Plasmodium falciparum (Pf) human life cycle ${ }^{8}$. In specific field trials, RTS,S-containing vaccines have been administered with either the $A S O 1$ or $A S O 2$ adjuvant systems ${ }^{8}$. By virtue of their intrinsic properties and composition, therefore, vaccines containing the RTS,S formulation impart partial pre-erythrocytic 


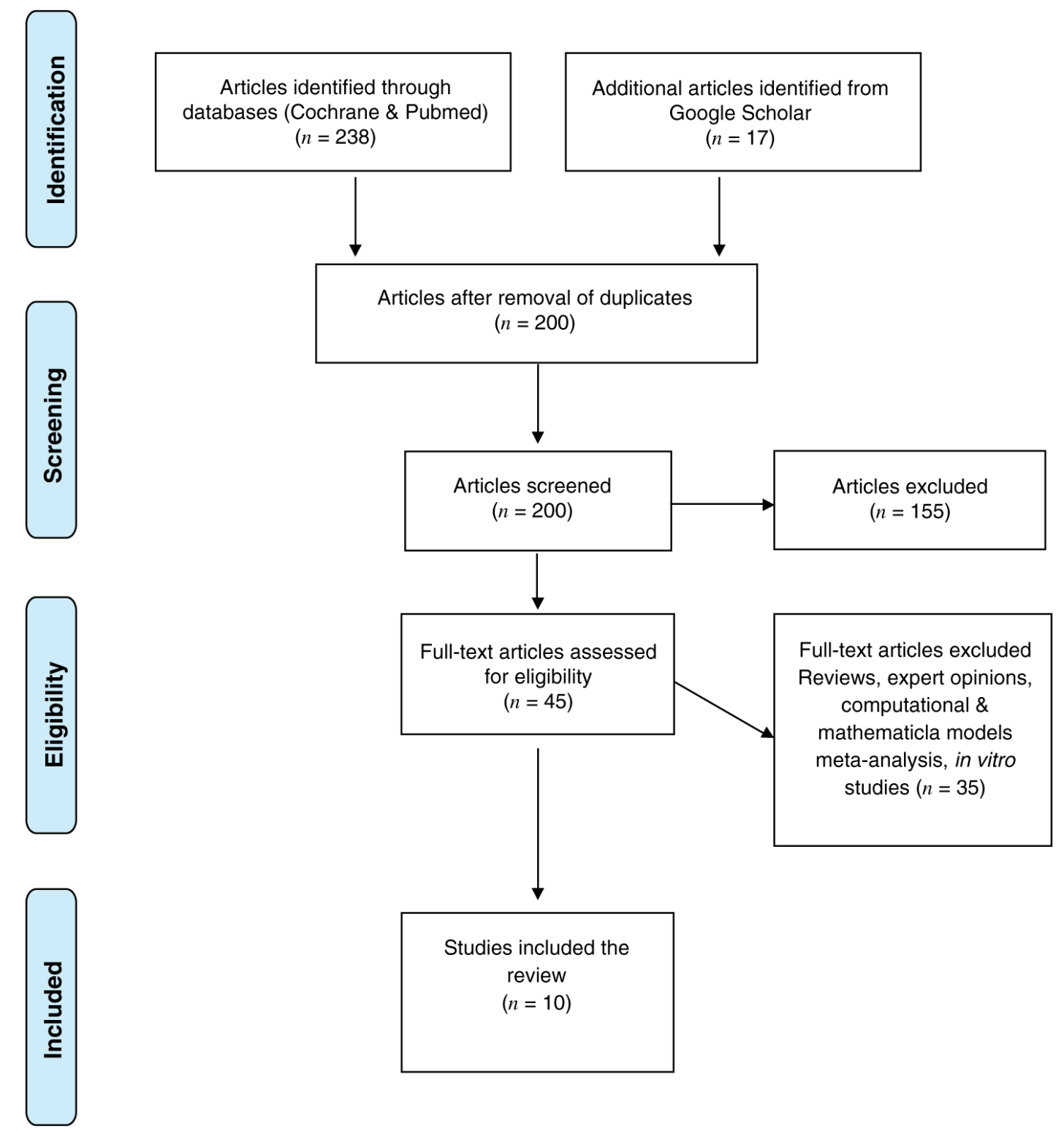

Figure 1. PRISMA flow diagram for screening inclusion and exclusion of studies.

immunity which is distinct from naturally-acquired immunity that mainly targets blood-stage parasites ${ }^{8,16}$. The latter type of immunity is hypothesized to be mediated mainly by T- and B-cell responses, as well as other possible mechanisms that are not presently well defined. An immuno-epidemiological study conducted by Bejon et al in 2011 concluded that vaccination of young children with the RTS,S/ASO1E vaccine resulted in reduced exposure to blood-stage parasites, therefore reducing anti-merozoite antigen antibody concentrations. The antibodies were, however, not correlates of clinical immunity to malaria ${ }^{8}$.
This is a serious concern associated with use of the RTS,S vaccine in the general population.

Several attempts have been made to improve the protective efficacy of the RTS,S vaccines. These include, but are not limited to, formulation with more potent adjuvant systems, use of boosted regimens with other CSP-expressing regimens and evaluation of other Pf antigens separately or in combination with $R T S, S$. An example of such a study was conducted using RTS,S combined with a recombinant form of thrombospondin 


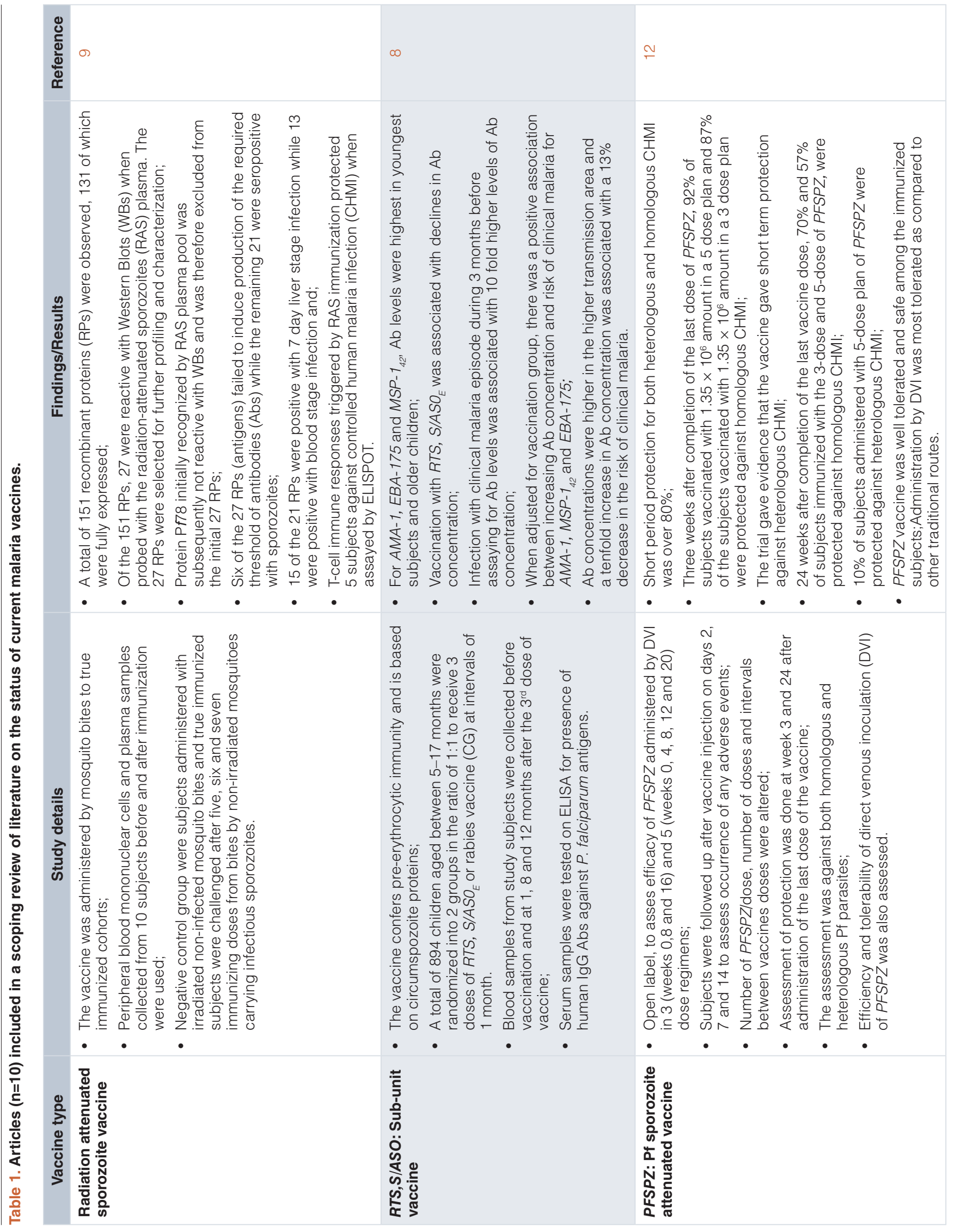




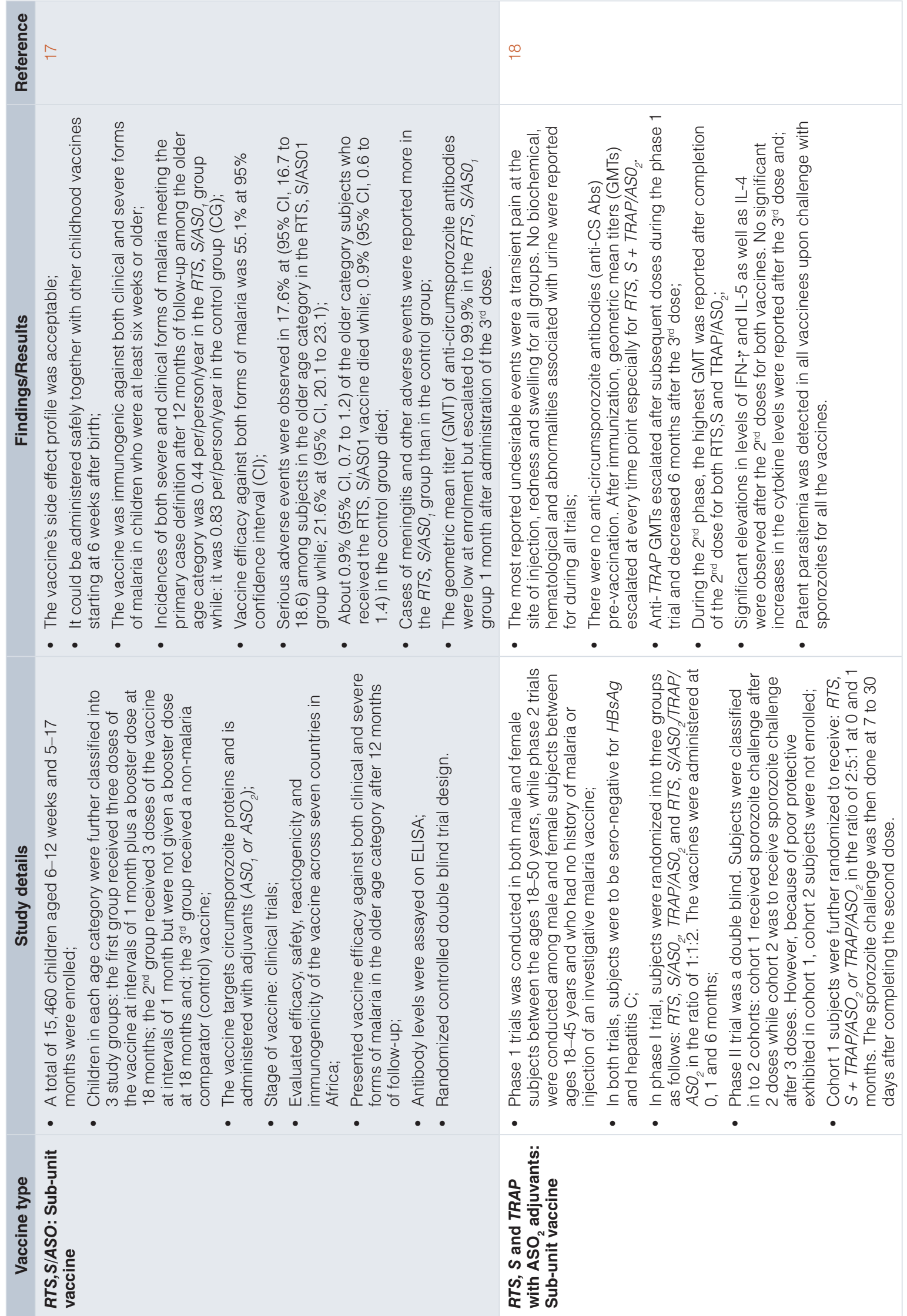




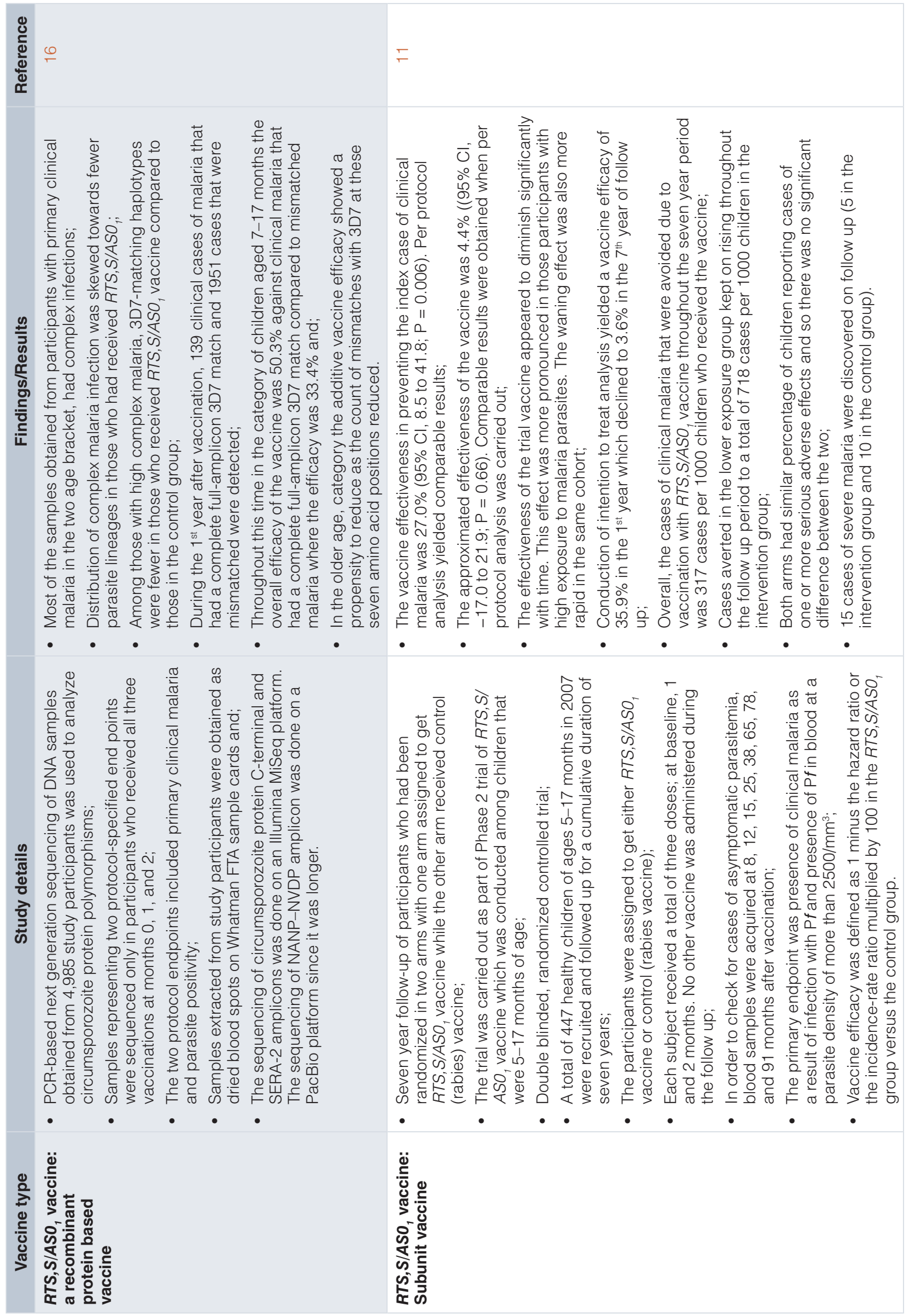




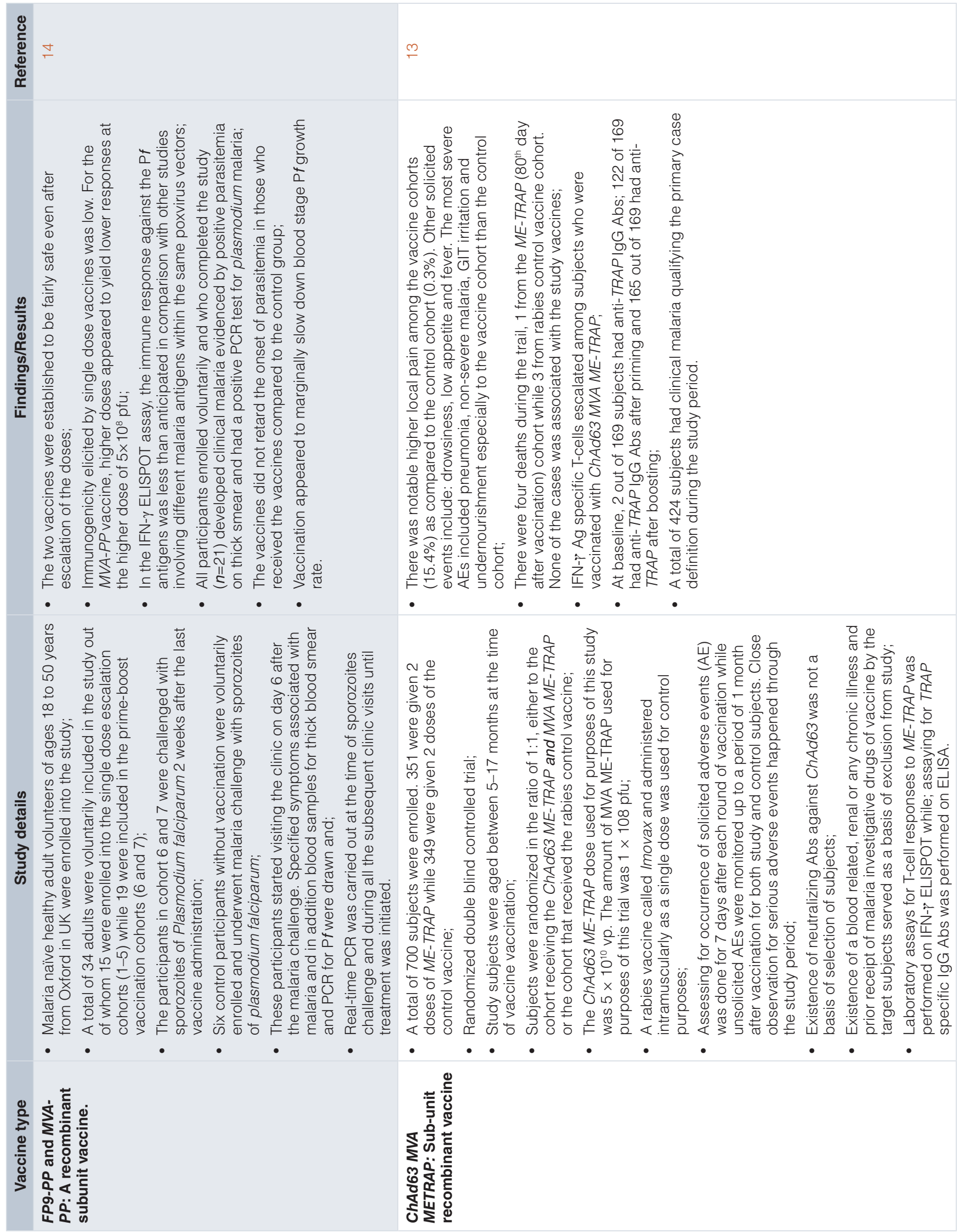




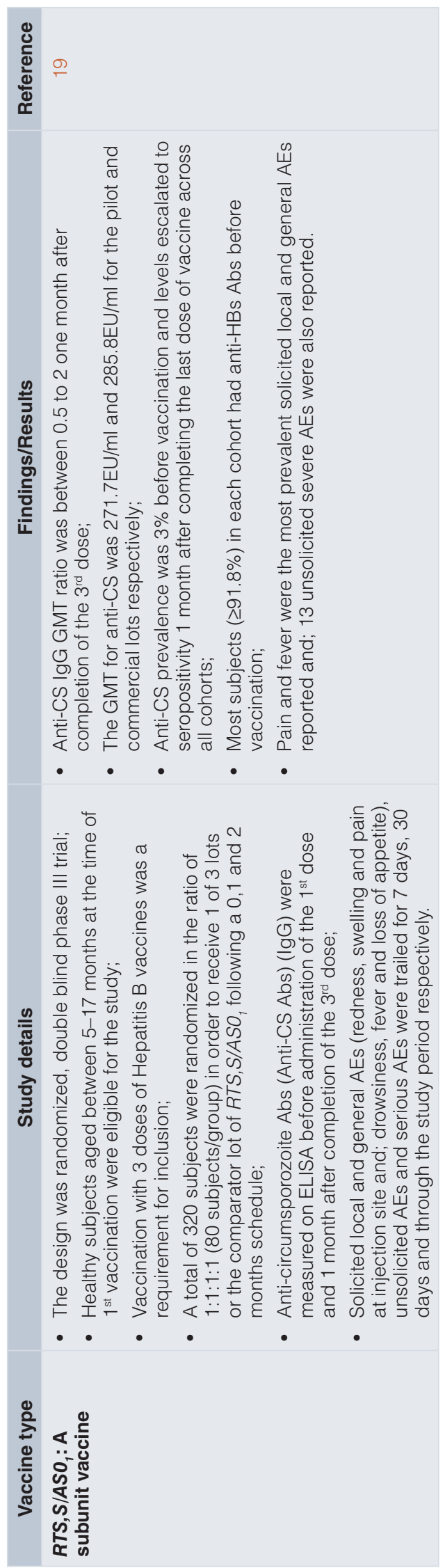


related anonymous protein $(T R A P)$ of Pf $(P f T R A P)^{18}$. Measurement of antigen-specific antibody responses, lymphoproliferative responses and IFN production due to the combination vaccine were similar to those produced by each of the single component vaccines. However, both the phase 1 and 2 clinical studies conducted by the investigators indicated inferior protective efficacy with the RTS,S/TRAP combination compared to single $R T S, S / A S O 2$ vaccine $^{18}$. This was attributed to possible immunological interference, a phenomenon whereby multiple antigens interfere with $\mathrm{T}$-cell priming and restimulation expected with a single antigen. The study was also limited by its significantly small sample size. Future combinations should perform preliminary exclusion of possible immunological interference between component antigens to improve success rates of such 'boosted' regimens.

An important consideration about use of the RTS,S vaccines is their duration of protective effect in the general population. Field trials of the vaccine have been conducted on infants and young children, where the vaccine has demonstrated average protection rates of between $18-36 \%$ with 3-4 doses of the vaccine administered during a 48-month follow-up period ${ }^{11}$. These trials were conducted in different African sites with varying rates of malaria transmission. Follow-up studies spanning a period of seven years however produced disappointing results on the long-term protective efficacy of the RTS,S malaria vaccine. From the two clinical studies, it was concluded that a threedose vaccination regimen with RTS,S/ASO1 vaccine offered initial protection against clinical malaria. The result was however offset in subsequent years, especially in areas with higher-than-average exposure to the parasites. Also, anti-merozoite antibody levels in vaccinated individuals were poor correlates of clinical immunity ${ }^{11}$.

A recent study investigating the relationship between protective efficacy of the RTS,S/ASO1 malaria vaccine and CSP polymorphisms in localized parasite population genotypes indicated that protective efficacy of the vaccine was higher in malaria parasites with matched CSP alleles than those with unmatched alleles $^{16}$. This finding is important because CSP polymorphisms and differences in haplotypic regions of the protein exert an effect of vaccine efficacy ${ }^{16}$. Significant differences were observed in the cumulative vaccine efficiency rate between different age groups, based on the CSP polymorphisms. Therefore, it may be helpful to incorporate CSP polymorphisms in the local parasite population in future vaccine development efforts. Such an expensive venture would definitely impact on the cost of production, and availability, of these tailor-made malaria vaccines.

On the bright side, a study investigating the immunogenicity and safety of commercially produced RTS,S/ASO1 malaria vaccine on a lot-to-lot basis yielded encouraging results. No significant variations were noted between the RTS,S/ASO1 vaccine lots formulated from a commercial-scale purified antigen bulk batch compared to those formulated from a pilot-scale antigen bulk batch ${ }^{19}$. This is an encouraging prospect for future production of multiple batches of the RTS,S pre-erythrocytic vaccines especially in resource-limited settings which are also malaria endemic.

A different approach from the RTS,S vaccine adopted in the last decade has led to development of the Pf sporozoite (PfSPZ) vaccine $^{12}$. This vaccine contains radiation-attenuated sporozoites of the parasite, and was assessed for tolerability, safety, immunogenicity and protective efficacy. The PfSPZ vaccine was administered by direct venous inoculation of 3-5 doses in non-immune individuals ${ }^{12}$. The $P f S P Z$ vaccine, given in a threedose regimen, protected against both 3- and 24-week homologous (similar strain as in vaccine) controlled malaria infections. No significantly alarming side effects were associated with the trialed regimens of this vaccine, and this is an encouraging outcome for future applications of the PfSPZ vaccine in mass malaria vaccination campaigns. Upcoming phase III trials of the vaccine will provide further insights on the protective efficacy and safety of the vaccine, especially against heterologous (different strain from vaccine) infections.

Furthermore, a polyprotein (PP) pre-erythrocytic malaria vaccine containing the entire sequence of six separate $P f$ proteins has been investigated. This vaccine was delivered using the combined viral vectors fowl pox virus strain FP9 and modified vaccinia virus Ankara $(M V A)^{14}$. The design strategy of this 'polyprotein' vaccine was targeted at producing a broad antibody response against a broad range of plasmodial pre-erythrocytic antigens, as opposed to a strong but narrow response. No serious adverse effects were observed with both the FP9-PP and $M V A-P P$ vaccines, but both failed to induce protection against sporozoite challenge or delay onset of parasitemia in vaccinated individuals ${ }^{14}$. The investigators attributed this outcome to possible limits in the size of immunogenic pre-erythrocytic proteins, at least when attached to viral vectors. Future work in the design of PP pre-erythrocytic malaria vaccines should therefore attempt to investigate the theory of size limits to immunogenicity, as well as effects of specific combinations and sizes to efficiency of the viral vectors employed. In addition, a separate phase IIa study utilizing the chimpanzee adenovirus 63 (ChAd63) and MVA vectors to express a TRAP-based vaccine yielded more promising results ${ }^{13}$. Both vaccines were demonstrated to be safe and adequately immunogenic, but induced only moderate $\mathrm{T}$-cell response. This immunogenic response was not sufficient to provide protection against clinical malaria in the follow-up period. Despite these results, viral vectors have a role in malaria vaccine delivery and future work should further investigate novel viral vectors as well as increased applicability in design of pre-erythrocytic vaccines.

Although CSP has stood out over the last few years as an immuno-dominant pre-erythrocytic antigen, several other proteins can be utilized in the design of pre-erythrocytic malaria vaccines. A study of 27 different pre-erythrocytic antigens indicated that 21 out of those, all localized to different parts of the sporozoite, induced detectable antibody responses in animal models9. These antigens elicited adequate antibody and T-cell responses and are therefore possible vaccine candidates. In 
future development of pre-erythrocytic malaria vaccines, thorough investigation of these additional sporozoite proteins should be undertaken to explore their viability as starting points for vaccine development. It would be interesting to explore these additional proteins as vaccine candidates.

This review had some limitations. We only considered vaccine candidates in clinical trials in the past decade. Therefore, promising vaccine candidates which have not yet been approved for clinical trials were not considered. In addition, only the vaccine candidates affecting the pre-erythrocytic stage were evaluated yet it is possible for a molecule affecting the erythrocytic stage to be an effective malaria vaccine. Articles published in another language apart from English were not included leading to exemption of potentially promising vaccine candidates.

\section{Conclusion}

The search for a malaria vaccine targeting the pre-erythrocytic stage of the Pf has led to development of sub-unit vaccines, for example, RTS,S vaccine \& ChAd63 MVA METRAP vaccine and inactivated vaccines like radiation attenuated Pf sporozoite vaccine. However, these vaccines have not been shown to induce sufficient immune responses required to provide adequate protection against malaria. The RTS,S vaccine currently undergoing clinical trials remains the most promising. However, its inability to confer long-term protection against clinical malaria and the varied antibody responses associated with the vaccine remain major drawbacks. The attenuated sporozoite vaccine is also a promising option, and ongoing clinical trials are vital in providing conclusions on the protective effect of this vaccine. Most importantly, ability of the PfSPZ vaccine to impart protection against heterologous malaria infections will be vital. Use of viral vectors for malaria vaccines has proven to be a viable mode of delivery. Further work is needed to identify optimal viral vectors for maximum protective efficacy. In addition, the possibility of using combined antigens in a single vaccine and further exploration of other plasmodial antigens as vaccine candidates are both routes that need to be pursued.

\section{Data availability}

Underlying data

All data underlying the results are available as part of the article and no additional source data are required.

\section{Reporting guidelines}

Harvard Dataverse: PRISMA-ScR checklist for 'Malaria vaccines targeting the pre-erythrocytic stage: a systematic review', https://doi.org/10.7910/DVN/ZK3ZZH ${ }^{20}$.

\section{Acknowledgements}

We acknowledge the technical input of Ms. Regina Njeru of the International Livestock Research Institute (ILRI). We are very grateful that she made time to give her valuable contribution.
1. World Health Organization World Malaria Report: World Health. WHO/HTM/GM 2010; 238.

Reference Source

2. Sultana M, Sheikh N, Mahumud RA, et al:: Prevalence and associated determinants of malaria parasites among Kenyan children. Trop Med Health. 2017; 45: 25 .

PubMed Abstract | Publisher Full Text | Free Full Text

3. Halliday KE, Okello G, Turner EL, et al.: Impact of Intermittent Screening and Treatment for Malaria among School Children in Kenya: A Cluster Randomised Trial. PLoS Med. 2014; 11(1): e1001594.

PubMed Abstract | Publisher Full Text | Free Full Text

4. West PA, Protopopoff N, Wright A, et al:: Indoor Residual Spraying in Combination with Insecticide-Treated Nets Compared to Insecticide-Treated Nets Alone for Protection against Malaria: A Cluster Randomised Trial in Tanzania. PLoS Med. 2014; 11(4): e1001630. PubMed Abstract | Publisher Full Text | Free Full Text

5. Coelho $\mathrm{CH}$, Doritchamou JYA, Zaidi I, et al.: Advances in malaria vaccine development: Report from the 2017 malaria vaccine symposium. npj Vaccines. 2017; 2: 34.

PubMed Abstract | Publisher Full Text | Free Full Text

6. Crompton PD, Pierce SK, Miller LH: Advances and challenges in malaria vaccine development. J Clin Invest. 2010; 120(12): 4168-78. PubMed Abstract | Publisher Full Text | Free Full Text

7. Duffy PE, Sahu T, Akue A, et al:: Pre-erythrocytic malaria vaccines: identifying the targets. Expert Rev Vaccines. 2012; 11(10): 1261-80. PubMed Abstract | Publisher Full Text | Free Full Text

8. Bejon P, Cook J, Bergmann-Leitner E, et al.: Effect of the pre-erythrocytic candidate malaria vaccine RTS,S/AS01E on blood stage immunity in young children. J Infect Dis. 2011; 204(1): 9-18.

PubMed Abstract | Publisher Full Text | Free Full Text
9. Aguiar JC, Bolton J, Wanga J, et al.: Discovery of novel plasmodium falciparum pre-erythrocytic antigens for vaccine development. PLoS One. 2015; 10(8): e0136109.

PubMed Abstract | Publisher Full Text | Free Full Text

10. Ndungu FM, Mwacharo J, Wambua J, et al:: A seven-year study on the effect of the pre-erythrocytic malaria vaccine candidate RTS,S/AS01 on blood stage immunity in young kenyan children [version 1; peer review: 1 approved, 2 approved with reservations]. Wellcome Open Res. 2019; 4: 42 . PubMed Abstract | Publisher Full Text | Free Full Text

11. Olotu A, Fegan G, Wambua J: Seven-Year Efficacy of RTS,S/AS01 Malaria Vaccine Among Young African Children N Engl J Med. 2016; 374(26): 2519-29. PubMed Abstract | Publisher Full Text | Free Full Text

12. Epstein JE, Paolino KM, Richie TL, et al:: Protection against Plasmodium falciparum malaria by PfSPZ Vaccine. JCl Insight. 2017; 2(1): 1-14. PubMed Abstract | Publisher Full Text | Free Full Text

13. Tiono AB, Nébié I, Anagnostou N, et al:: First field efficacy trial of the ChAd63 MVA ME-TRAP vectored malaria vaccine candidate in 5-17 months old infants and children. PLOS One. 2018; 13(12): e0208328. PubMed Abstract | Publisher Full Text | Free Full Text

14. Porter DW, Thompson FM, Berthoud TK, et al:: A human phase I/lla malaria challenge trial of a polyprotein malaria vaccine. Vaccine. 2011; 29(43): 7514-22.

PubMed Abstract | Publisher Full Text | Free Full Text

15. Shamseer L, Moher D, Clarke M, et al:: Preferred reporting items for systematic review and meta-analysis protocols (prisma-p) 2015: Elaboration and explanation. BMJ. 2015; 350: g7647. PubMed Abstract | Publisher Full Tex

16. Neafsey DE, Juraska M, Bedford T, et al:: Genetic diversity and protective efficacy of the RTS,S/AS01 malaria vaccine. N Engl J Med. 2015; 373: 
2025-2037

Publisher Full Text

17. Agnandji ST, Lell B, Soulanoudjingar SS, et al: First results of phase 3 trial of RTS,S/AS01 malaria vaccine in African children. N Engl J Med. 2011; 365(20): $1863-1875$.

PubMed Abstract | Publisher Full Text

18. Kester KE, Gray Heppner D, Moris P, et al.: Sequential Phase 1 and Phase 2 randomized, controlled trials of the safety, immunogenicity and efficacy of combined pre-erythrocytic vaccine antigens RTS,S and TRAP formulated with AS02 Adjuvant System in healthy, malaria naïve adults. Vaccine. 2014; 32(49):
6683-6691.

PubMed Abstract | Publisher Full Text

19. Umeh R, Oguche S, Oguonu T, et al:: Immunogenicity and safety of the candidate RTS,S/AS01 vaccine in young Nigerian children: A randomized, double-blind, lot-to-lot consistency trial. Vaccine. 2014; 32(48):

6556-6562.

PubMed Abstract | Publisher Full Text

20. Walekhwa M: Malaria vaccines targeting the pre-erythrocytic stage: a systematic review. Harvard Dataverse, V3. 2020.

http://www.doi.org/10.7910/DVN/ZK3ZZH 


\section{Open Peer Review}

\section{Current Peer Review Status: $\mathrm{X} X$}

\section{Version 1}

Reviewer Report 07 October 2021

https://doi.org/10.5256/f1000research.26833.r96331

(c) 2021 Prudêncio $M$. This is an open access peer review report distributed under the terms of the Creative Commons Attribution License, which permits unrestricted use, distribution, and reproduction in any medium, provided the original work is properly cited.

\section{Miguel Prudêncio}

Faculty of Medicina, Instituto de Medicina Molecular João Lobo Antunes, University of Lisboa, Lisboa, Portugal

The authors set out to write a "scoping review" of pre-erythrocytic malaria vaccines. However, this manuscript does not even remotely cover all relevant aspects of pre-erythrocytic vaccination against malaria. This is hardly surprising, when the authors themselves claim to have reviewed "ten papers" to write this review, when there are hundreds of papers on this subject, and many dozens of these include results on the clinical evaluation of pre-erythrocytic malaria vaccine candidates. It is not even clear how these ten papers were selected, let alone the fact that it is very arguable that they are all among the most relevant in the field. For example, there are no references to genetically attenuated sporozoites (GAS), sporozoites administered under chemoprophylaxis (CPS), Sanaria's PfSPZ-CVAC, a newly described strategy based on genetically modified $P$. berghei parasites expressing P. falciparum antigens, or the new subunit vaccine candidate R21 developed by the University of Oxford, all of which have been tested in the clinic. This places this manuscript a very long way from being considered "a scoping review".

Besides the evident absence of many references to crucial work in this field, the manuscript is also fraught with incorrections or misleading remarks, including in the abstract, such as "transmission blocker (sic) vaccines ... only protect the immunized population" (vaccines usually aim to protect those immunized; what the authors probably meant is that transmission-blocking vaccines are altruistic vaccines that only work at the community level) and "Vaccines targeting blood-stage forms... are challenged by the complex metabolic pathways of erythrocytes and merozoites" (I would argue that the challenges for blood stage vaccines go well beyond the metabolism of erythrocytes and merozoites). Overall, the article falls very short of its stated objective and, in my opinion, does not meet the minimal standards for indexing.

\section{Are the rationale for, and objectives of, the Systematic Review clearly stated?} Partly

Are sufficient details of the methods and analysis provided to allow replication by others? 
No

Is the statistical analysis and its interpretation appropriate?

Not applicable

Are the conclusions drawn adequately supported by the results presented in the review? Partly

Competing Interests: No competing interests were disclosed.

Reviewer Expertise: Pre-erythrocytic stage of Plasmodium infection; Malaria vaccines; Antiplasmodial therapeutics

I confirm that I have read this submission and believe that I have an appropriate level of expertise to state that I do not consider it to be of an acceptable scientific standard, for reasons outlined above.

Reviewer Report 26 October 2020

https://doi.org/10.5256/f1000research.26833.r71931

(C) 2020 Burns Jr J. This is an open access peer review report distributed under the terms of the Creative Commons Attribution License, which permits unrestricted use, distribution, and reproduction in any medium, provided the original work is properly cited.

\section{James M Burns Jr}

Department of Microbiology and Immunology, Center for Molecular Parasitology, Drexel University College of Medicine, Philadelphia, Pennsylvania, USA

In this manuscript, Ogeto et al attempt to provide a comprehensive review of malaria vaccines targeting pre-erythrocytic stages of Plasmodium falciparum. The review falls short of achieving that goal. Although the period from 2010-2020 was considered, only 10 publications were evaluated. The criteria for selection of these articles was not adequately presented. For example 155 articles were deemed not eligible by two reviewers, but the basis for exclusion was not clear. The bulk of the article is Table 1 which simply provides a basic description of each study and a list of findings. In some cases, the information is not adequate to understand the composition of each vaccine formulation and/or to appreciate the primary goal of each study. Overall, the review lacked depth, breadth and a clear theme for an integrated comparison of the papers selected. Considering published reviews covering related topics (i.e. progress on malaria vaccine development, RTS,S, whole sporozoite vaccines), there was little new information or insight provided by the authors. The effort could benefit from a more thorough, critical evaluation of clinical trial results. It is not clear that this summary of pre-erythrocytic malaria vaccine trials will be of substantive value to the field.

In places, the point the authors were trying to convey was not clear. For example, the basis for the following statements is not apparent and/or is confusing: 
Abstract: "Vaccines targeting blood-stage forms, such as AMA-1 and MSP-1 vaccines, are challenged by the complex pathways of erythrocytes and merozoites."

Introduction: "Their superiority is founded primarily on the premise that sporozoites harbor less antigens and are thus easily targeted by an appropriate vaccine. This results in reduced merozoite production thereby rendering the blood stage immune responses superior".

Are the rationale for, and objectives of, the Systematic Review clearly stated?

Yes

Are sufficient details of the methods and analysis provided to allow replication by others? No

Is the statistical analysis and its interpretation appropriate?

Not applicable

Are the conclusions drawn adequately supported by the results presented in the review? No

Competing Interests: No competing interests were disclosed.

Reviewer Expertise: malaria vaccines

I confirm that I have read this submission and believe that I have an appropriate level of expertise to state that I do not consider it to be of an acceptable scientific standard, for reasons outlined above.

The benefits of publishing with F1000Research:

- Your article is published within days, with no editorial bias

- You can publish traditional articles, null/negative results, case reports, data notes and more

- The peer review process is transparent and collaborative

- Your article is indexed in PubMed after passing peer review

- Dedicated customer support at every stage

For pre-submission enquiries, contact research@f1000.com 